\title{
Homologous integration in mammalian cells without target gene selection
}

\author{
Maria Jasin and Paul Berg \\ Department of Biochemistry, Stanford University Medical Center, Stanford, California 94305 USA
}

\begin{abstract}
Homologous integrations into a nonselectable target locus have been highly enriched for following DNA transfections into mammalian cells. The target gene, the SV40 early region in COS1 cells, provides transcription signals to activate a defective selectable marker, the gpt gene. We find that nearly half of the selected clones have integrated the gpt gene at the homologous sequence in the COS1 genome. This is an estimated 100-fold enrichment for homologous events compared with transfections in which the gpt gene is transcriptionally active. As shown for yeast integration events, a double-strand break at a position of homology between the transfected DNA and the genomic target is necessary to achieve a high frequency of homologous integrations. Furthermore, the arrangement of sequences at the integration site includes a repair of the doublestrand gap, which was present on the transfected DNA, suggesting that similarities exist between yeast and mammalian integrations. The experimental design, in which a defective marker is activated following a homologous integration, may have general applications for gene targeting in mammalian cells.
\end{abstract}

[Key Words: Recombination; COS1 cells; $\mathrm{T}$ antigen; double-strand gap repair]

Received July 19, 1988; revised version accepted September 6, 1988.

Reverse genetic studies of cloned genes in prokaryotes and yeast have relied heavily on homologous recombination between transfected and genomic DNA sequences. However, in mammalian systems, the preponderant mode of recombination between transfected and genomic DNAs involves nonhomologous sequences. The reasons for this difference are not known. If homologous recombination in mammalian cells could be enhanced relative to the nonhomologous reactions, or if the two kinds of events could be distinguished more efficiently, new opportunities for studies in mammalian genetics would be created and questions concerning the regulation and expression of genes could be addressed more precisely. Targeted integrations of transfected DNAs would make it possible to mutagenize genetic loci of cultured cells and eventually of whole animals such as mice, and the correction of inherited human defects might follow.

Homology-directed integrations in mammalian systems have been detected in two ways. In the first, cells are subjected to a positive or negative selection to isolate the homologous recombinants (Smith and Berg 1984; Lin et al. 1985; Thomas et al. 1986; Doetschman et al. 1987; Song et al. 1987; Thomas and Capecchi 1987). In a completely different approach, recombinants are identified through extensive screening procedures (Smithies et al. 1985). The first approach is not very general, as very few genes can be selected easily for or against, especially in the diploid state. The second approach, which is very cumbersome, can, nevertheless, be applied to any cloned gene. In this paper, an alternative approach to detect homology-directed integrations in mammalian cells is described. The target gene provides signals for the proper expression of a defective selectable marker. The nonhomologous background is lowered, effectively enriching for the homologous events.

Previously, we had used the SV40 early region transcription unit, which is stably integrated in COS cells (Gluzman 1981), as the genomic target for recombination (Jasin et al. 1985). Transfection of COS1 cells with SV40 DNA lacking the transcription enhancer leads to the production of enhancer-containing wild-type virus. These experiments indicated that the enhancer can be acquired in a nonintegrative homologous recombination between transfected DNA and a genomic target. Similar experiments involving plasmid/chromosomal recombination in yeast had shown that integrative and nonintegrative events are equally probable (Orr-Weaver and Szostak 1983). It seemed possible that integration of the transfecting viral DNA might also occur in the SV40 system but not be detected because of viral replication and subsequent cell lysis during the viral infection. To examine this possibility, the experimental design was modified to allow efficient detection of homologous integrations. As before, the target gene, the SV40 early region, contributes the transcription enhancer (and in some cases, promoter sequences) to the transfected DNA; however, in this case, the transfected DNA is a transcriptionally defective selectable marker, the gpt gene (Mulligan and Berg 1981), linked to SV40 sequences. Homologous recombinants, in which the gpt gene acquires the missing transcription signals via integration at the target sequence, are readily selected in media containing mycophenolic acid. Using this experi- 
mental design, we find that nearly half of the mycophenolic-acid-resistant clones have integrated the gpt gene at the homologous sequence in the SV40 early region. The remaining mycophenolic-acid-resistant clones arise from nonhomologous integration of the transfected plasmid. Because the transfected gpt gene is defective, the number of random integrations detected is $\sim 100$ fold lower than the number that occurs when the transfected gene is transcriptionally active.

Further results from these experiments are similar to those obtained in yeast integration events. A doublestrand break at a position of homology between the transfected DNA and the genomic target is necessary to achieve a high frequency of homologous integrations. Furthermore, the arrangement of sequences at the integration site includes a repair of the enhancer deletion present on the transfected DNA. Both of these observations are consistent with recombination occurring by the double-strand break repair mechanism as proposed by Szostak et al. (1983). Additionally, the target gene can be deleted in a secondary homologous recombination analogous to the two-step gene replacements in yeast (Scherer and Davis 1979).

\section{Results}

\section{Design of the recombination substrates}

The array of SV40 sequences in COS1 cells, the structure of two integrating plasmids, and the predicted arrangement of sequences following homologous integration of each are summarized in Figure 1A. COS1 cells contain the SV40 early region transcription unit integrated at a single site in their genomic DNA /Gluzman 1981). This copy contains the transcription enhancer $(E)$, promoter $(\mathrm{P})$, and the coding sequence for the $\mathrm{T}$ antigens (early). The integrated copy is stable because the origin of replication $(\mathrm{O})$ contains a small deletion rendering it replication defective. Although the SV40 early region occurs at only one chromosomal site, the number of copies appears to be variable, possibly due to aneuploidy or amplification of the site. Adjacent to the early region is a contiguous portion of SV40 DNA encoding part of the late region.

The integrating plasmid $\Delta 1$ has the gpt gene derived from the plasmid pSV2gpt (Mulligan and Berg 1980). The gpt-coding sequence is flanked upstream by the SV 40 early promoter and downstream by the SV40 small $\mathrm{t}$ antigen intron and polyadenylation site (see below; Fig. 4B). The SV40 origin of replication contains the same deletion as the chromosomal copy; but, most importantly, the promoter lacks the transcription enhancer, thus rendering the gpt gene transcriptionally impaired. The transfecting and genomic DNA share homology in the sequences that flank the enhancer. The homology extends for $1.3 \mathrm{~kb}$ from the late region through $\mathrm{PO}$ and is interrupted by the 166-bp enhancer deletion. The bacterial vector sequences in $\Delta 1$ (pUC19 not shown) reside between the late region and the promoter, but are cleaved away prior to transfection. Integrating plasmid $\Delta 2$ is identical to $\Delta 1$ except that the vector segment occurs between the late region and the end of the gpt transcription unit.

The transfecting DNAs had a double-strand break at the enhancer deletion, thereby producing a doublestrand gap at this position relative to the genomic target. According to the double-strand break repair model (Szostak et al. 1983), homologous integration would create a duplication of $\mathrm{E}$ flanking the integrating plasmid. This would result in an activation of transcription of gpt without disnupting transcription of the target gene. The selection for gpt relied on the expectation that homologous recombinants would be selected preferentially because most nonhomologous integrations would not activate gpt expression unless the gpt gene fortuitously integrated into an actively transcribing locus or at high copy number. Maintenance of the $T$ antigen sequences was desirable in the event that loss of $T$ antigen expression might confer a selective disadvantage to the desired recombinants.

\section{Efficient homologous integrations with $\Delta 1$ and $\Delta 2$}

The integrating plasmids $\Delta 1$ and $\Delta 2$, cleaved at the enhancer deletion, were transfected independently into COS1 cells by the calcium phosphate precipitation method. Two days post-transfection, the cells were split to a density of $3 \times 10^{5}$ or $6 \times 10^{5}$ cells per $10-\mathrm{cm}$ plate, and, after another day, selective medium containing mycophenolic acid was added. A frequency of $\sim 1 \times 10^{-5}$ to $2 \times 10^{-5} \mathrm{gpt}^{+}$colonies per total number of cells was obtained by transfection of cleaved $\Delta 1$ and $\Delta 2$ (Table 1A). Transfection with circular plasmid $2 \mathrm{E}$, which contained the SV40 enhancer, produced $g p t^{+}$transformants at a frequency 100 -fold higher.

Homologous integrations were identified by Southern blot analysis of the DNA obtained from mycophenolicacid-resistant clones. Blots were hybridized initially with an early region probe specific for the genomic target to determine whether there had been a change in the sequence arrangement at the chromosomal site. Subsequently, the blots were hybridized with a probe containing gpt sequences to determine whether the sequence alteration was associated with the acquisition of the gpt gene.

Figure 1. Scheme for homologous integrations in COS1 cells. (A) The genomic target for integration, the SV40 locus in COS1 cells, is diagramed on the central line. Homologous integrations of plasmids $\Delta 2$ and $\Delta 1$ are predicted to give the arrangement of sequences shown on the upper and lower portions of the figure, respectively. Integrations are drawn with concomitant repair of the enhancer deletion, resulting in a duplication of the enhancer. The plasmids are introduced with a double-strand break at the position of the enhancer deletion. $(B)$ Homologous integrations of plasmids $\Delta 3-\Delta 5$, as in A. (EPO) SV40 control region elements; (E) transcription enhancer; $(\mathrm{P})$ additional promoter elements; $\left(\mathrm{O}^{\Delta}\right)$ defective origin of replication. The $\mathrm{E}$ deletion for $\Delta 1-\Delta 3$ is $166 \mathrm{bp}$. The EPO deletion for $\Delta 4$ and $\Delta 5$ is $344 \mathrm{bp}$. Each of the plasmids has $1.1 \mathrm{~kb}$ of homology with the genome at the late region sequences. The homology with the genome extends for $194 \mathrm{bp}$ at $\mathrm{PO}$ in $\Delta \mathrm{l}$ and $\Delta 2,807 \mathrm{bp}$ at the PO/early region segment in $\Delta 3$, and $663 \mathrm{bp}$ at the early region in $\Delta 4$ and $\Delta 5$. 


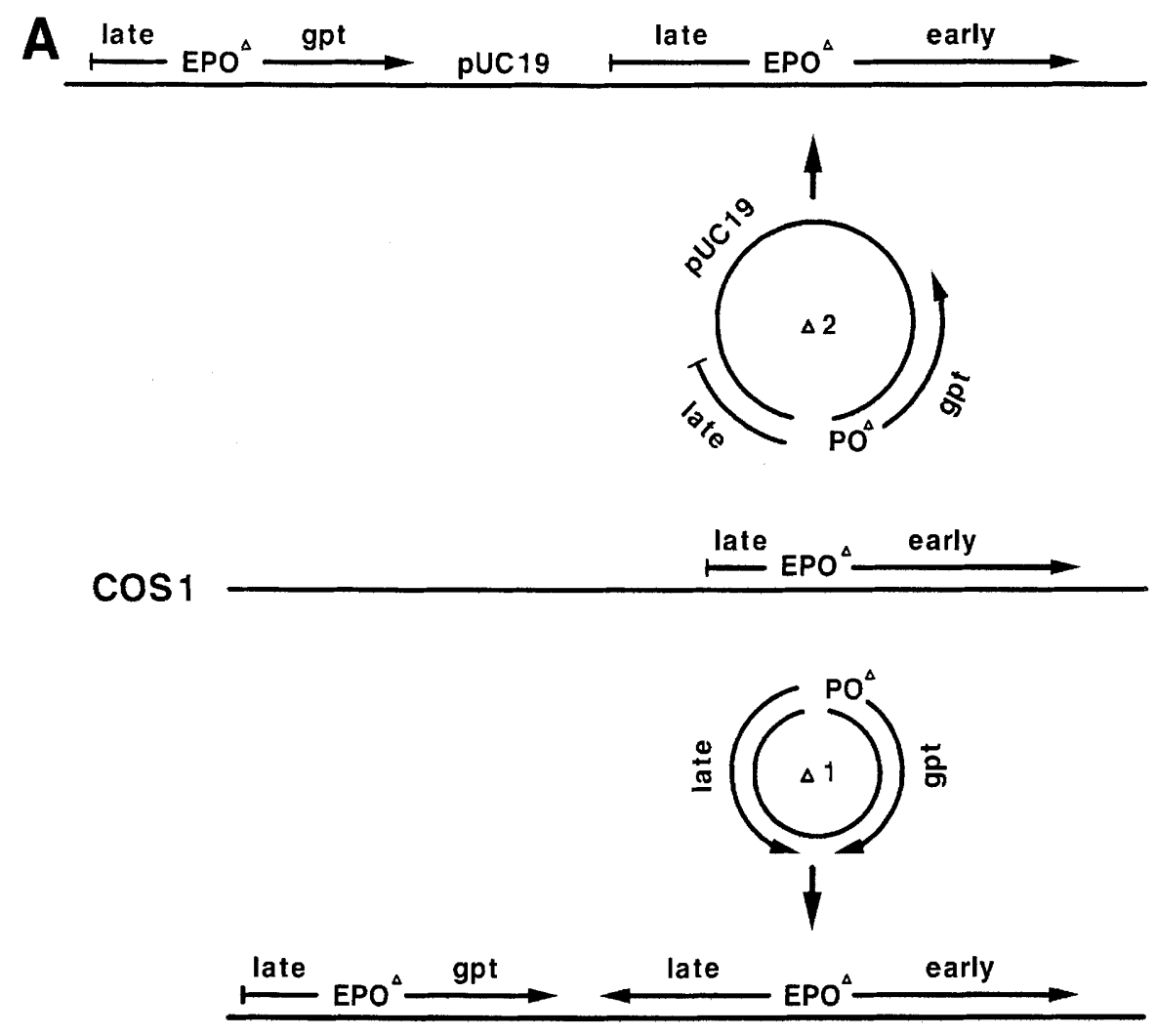

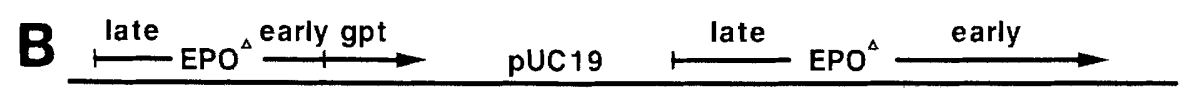
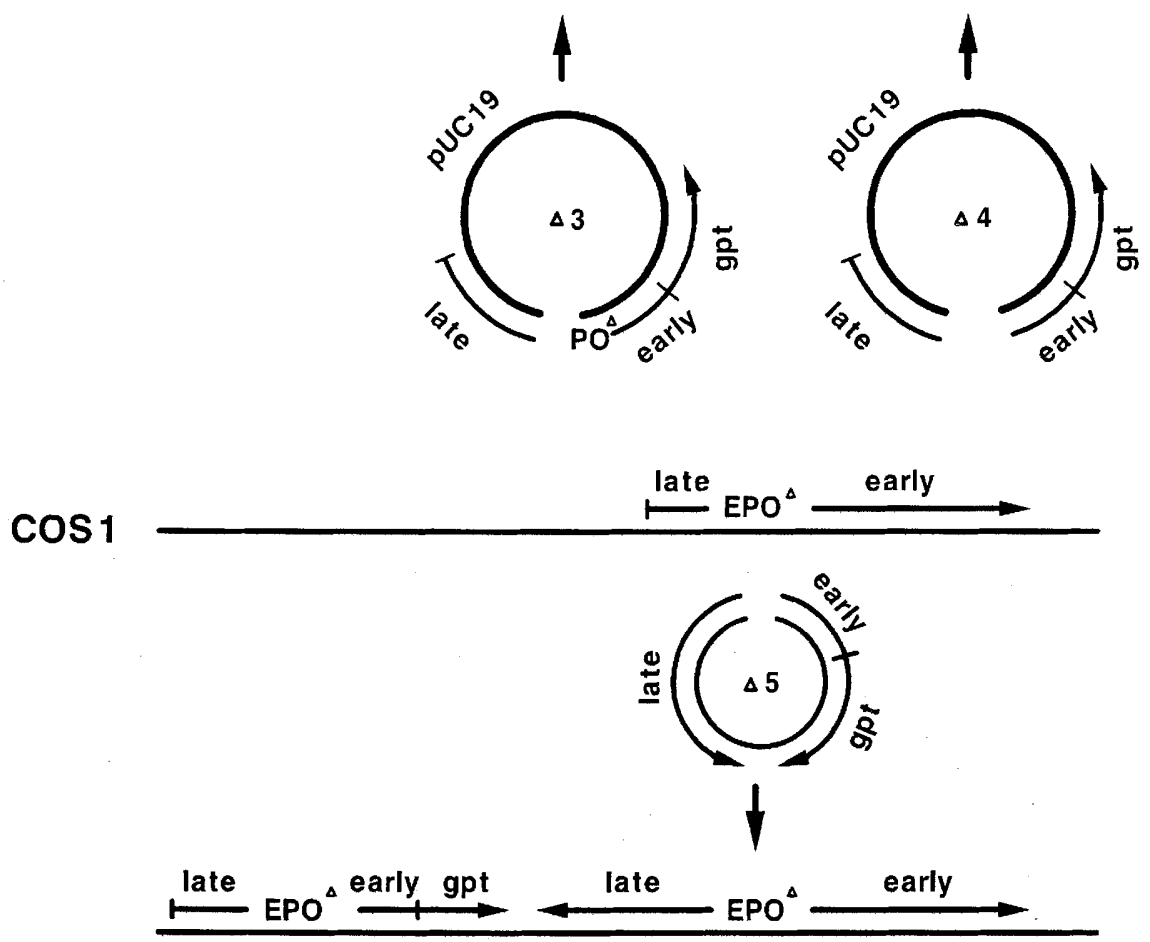

Figure 1. (See facing page for legend.) 
Table 1. Efficient homologous integration with defective gpt plasmids

\begin{tabular}{|c|c|c|c|}
\hline $\begin{array}{l}\text { Input } \\
\text { DNA }\end{array}$ & $\begin{array}{c}\text { Transformation } \\
\text { efficiency }\end{array}$ & $\begin{array}{l}\text { Homologous } \\
\text { recombinants }\end{array}$ & $\begin{array}{c}\text { Fraction } \\
\text { homologous }\end{array}$ \\
\hline \multicolumn{4}{|c|}{$A$} \\
\hline$\Delta \mathrm{l}$ & $2 \times 10^{-5}$ & $9 / 18$ & 0.50 \\
\hline$\Delta 2$ & $1 \times 10^{-5}$ & $7 / 9$ & 0.78 \\
\hline $2 \mathrm{E}$ & $1 \times 10^{-3}$ & N.D. & - \\
\hline \multicolumn{4}{|c|}{$B$} \\
\hline$\Delta 3$ & $5 \times 10^{-5}$ & 19 & 0.11 \\
\hline$\Delta 4$ & $5 \times 10^{-5}$ & $6 / 37$ & 0.16 \\
\hline$\Delta 5$ & $4 \times 10^{-5}$ & $8 / 33$ & 0.24 \\
\hline $3 \mathrm{E}$ & $3 \times 10^{-4}$ & N.D. & - \\
\hline
\end{tabular}

The transformation efficiency is the ratio of the number of $g p t^{+}$ clones to the total number of COS1 cells plated at the time selection was imposed. Transformants were screened by BglII$B c l l$ restriction and Southern blot analysis and scored as homologous recombinants if the shifted endogenous early region band was associated with hybridization of the gpt gene. Plasmids $2 \mathrm{E}$ and $3 \mathrm{E}$ contain a complete SV40 enhancer/promoter region but are otherwise identical to plasmids $\Delta 2$ and $\Delta 3$, respectively. Plasmids $\Delta 1-\Delta 5$ were introduced with double-strand breaks at the enhancer deletion; plasmids $2 \mathrm{E}$ and $3 \mathrm{E}$ were introduced uncut. The data for homologous recombinants for plasmid $\Delta 1$ were derived from two independent platings of cells; for $\Delta 2$ and $\Delta 3$, one plating; for $\Delta 4$ and $\Delta 5$, four platings. (N.D.) Not determined.

The first digest chosen for this analysis was made with BglII and BclI endonucleases (Fig. 2). COSI DNA, when hybridized with the early region probe, yields a 4.8-kb fragment defined by the BclI endonuclease cleavages. If the $\Delta 1$ plasmid had undergone homologous recombination at this position, the fragment would be $6.8 \mathrm{~kb}$ in length, the distance between the the BclI restriction site in the endogenous early region and BgIII cleavage site in the gpt sequence. For $\Delta 2$, the fragment would be expected to be $9.5 \mathrm{~kb}$, due to the presence of vector sequences. Both the 6.8- and 9.5-kb fragments should also hybridize to the gpt probe. If the gpt plasmid had integrated at nonhomologous loci, the structure of the SV40 early region should have remained unchanged and the fragments hybridizing to the gpt probe would have varied in size depending on the distance from the $B g I I I$ restriction site in $g p t$ to flanking $B g I I I$ or $B c l I$ sites in the chromosomal DNA.

Southern blots of DNA from several $\Delta 1$ and $\Delta 2$ derived $\mathrm{gpt}^{+}$transformants are shown in Figure 2. The clones whose band patterns contain either a 6.8 - or a $9.5-\mathrm{kb}$ band (for $\Delta 1$ and $\Delta 2$, respectively), when hybridized with both the early region probe and the gpt probe, are indicated by asterisks. Other clones have an unaltered early region band and variable-sized gpt bands, indicative of nonhomologous integrations. These results and the analysis of Southern blots using other restriction enzymes (data not shown) are summarized in Table 1A. Of the $18 \mathrm{gpt}^{+}$transformants resulting from transfection with plasmid $\Delta 1$, nine appear to be homologous recom- binants. Several of these nine clones (e.g., clone $\Delta 1 \mathrm{~B}-1$ ) retained the endogenous 4.8 -kb early band, indicating either that there was a second copy of the early region in the parental cell or that a second copy was generated during recombination. Some of the $g p t^{+}$clones also have a $4.6-\mathrm{kb}$ band that hybridizes with the gpt probe. Additional Southern blot analyses of digests with $B c$ lI endonuclease alone suggest that this band is due to integration of tandem copies of the transfecting gpt plasmid. Of the remaining nine clones, eight had only the endogenous 4.8 -kb early region band and variable-sized gpt bands. The ninth clone, $\Delta 1 \mathrm{~A}-1$, had an additional early region hybridizing band of $\sim 3 \mathrm{~kb}$, the origin of which has not been determined.

With plasmid $\Delta 2$, six out of nine $g p t^{+}$transformants had fragment patterns consistent with homologous integrations. The clone $\Delta 2 A-7$ appears to have a tandem integration of the $\Delta 2$ plasmid. A seventh clone $(\Delta 2 \mathrm{~A}-11$, not shown) has a $B g l I I-B c l$ I band that hybridizes with both the early region and gpt probes but is larger than the expected $9.5 \mathrm{~kb}$, suggesting an aberrant homologous integration.

Many of the homologous recombinants have very simple Southern blot patterns, i.e., a shift in the size of the early region fragment due to integration of a single copy of the transfected plasmid (e.g., clones $\Delta \mathrm{lA}-3$ and $\Delta 2 A-1)$. Many others, however, have more complex patterns, apparently due to either a tandem integration of the transfected plasmid or the presence of a second copy of the target gene (e.g., clone $\Delta 1 \mathrm{~B}-1$ has both of these features). We notice no correlation between the complexity of the pattern and the transfected plasmid used in 25 homologous recombinants obtained from two independent experiments (Table $1 \mathrm{~A}$ and data not shown). In none of these homologous recombinants is there any indication of additional, nonhomologous gpt integrations.

The double-strand gap repair model predicts that the enhancer deletion in the gpt promoter of the transfected DNA is repaired by the recombination (see Fig. 1). To test this expectation, DNA from the putative homologous recombinants obtained in the transfections with $\Delta 1$ and $\triangle 2$ DNAs was digested with $E c o R V$ endonuclease (Fig. 3). The predicted sizes of the fragments produced from the endogenous early region promoter and the reconstructed gpt promoter are 3.7 and $1.4 \mathrm{~kb}$, respectively. The data show that each of the DNAs obtained from the putative homologous recombinant clones yields the expected two fragments, a finding that is consistent with the double-strand gap repair model. Clone $\triangle 2 \mathrm{~A}-11$, an aberrant homologous recombinant according to the BgIII-BcII analysis, has an additional band.

Confirmation of the sequence arrangement shown in Figure 1 was obtained by cloning the integration site from two of the recombinants. Chromosomal DNA from recombinant clones $\Delta 2 \mathrm{~A}-1$ and $\triangle 2 \mathrm{~A}-3$ was cleaved with $B c l$ l endonuclease (see Fig. 2) and electrophoresed on a preparative agarose gel. DNA in the $12-\mathrm{kb}$ size range, determined by Southern analysis to contain the integration site, was recovered and ligated under dilute conditions. 
Cos1 - early probe-4.8 kb band; gpt probe-no hybridizing band

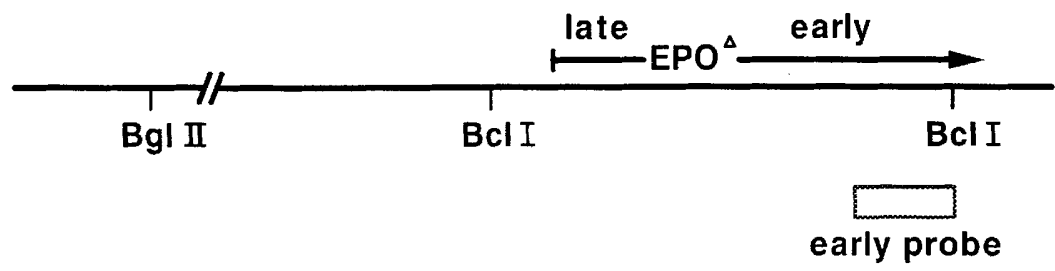

$\triangle 1$ HOMOLOGOUS INTEGRANT - early and gpt probes $-6.8 \mathrm{~kb}$ band

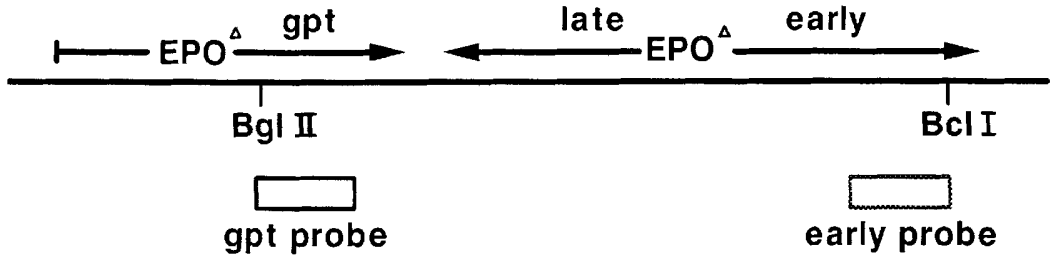

\section{$\triangle 2$ HOMOLOGOUS INTEGRANT - early and gpt probes $=9.5 \mathrm{~kb}$ band}
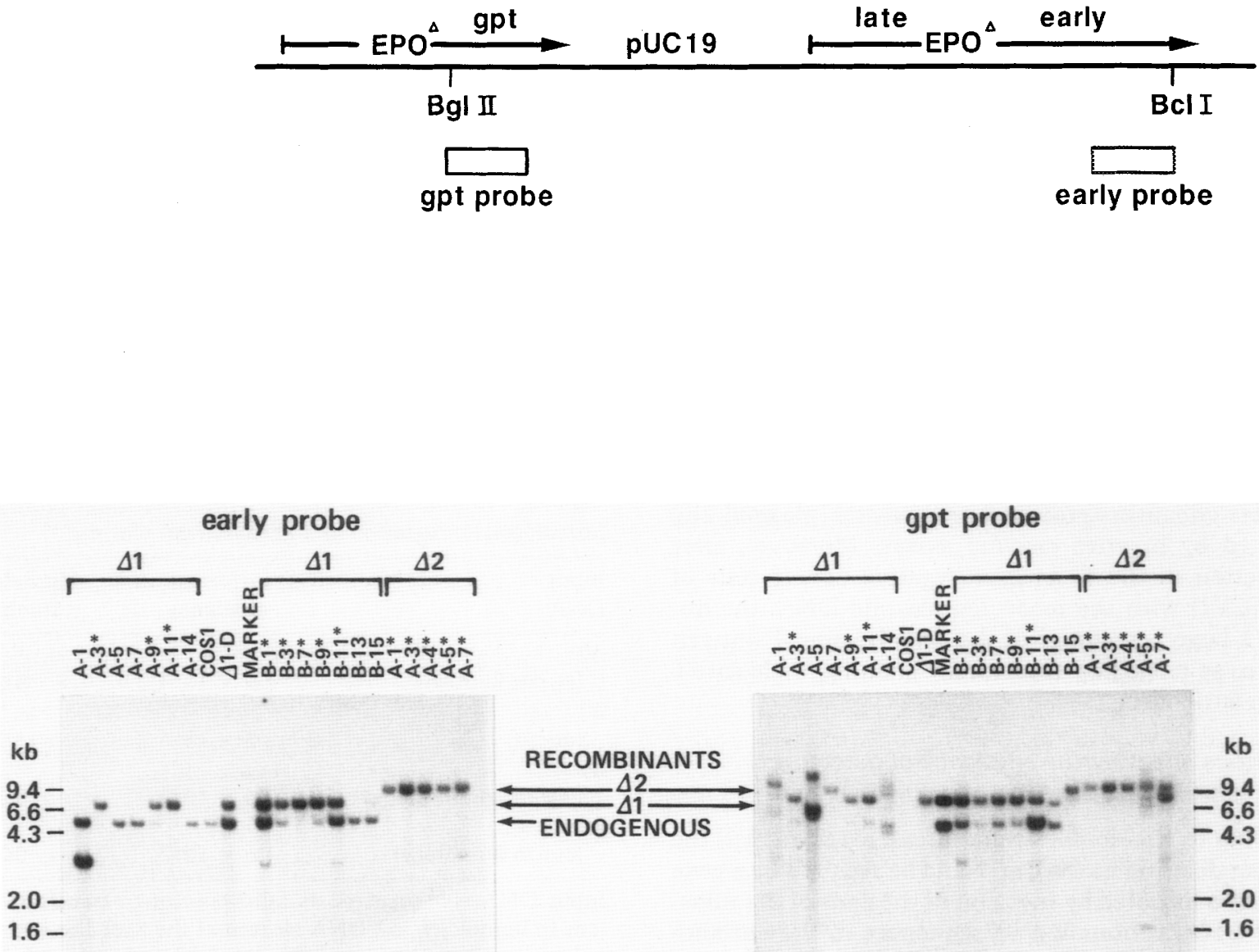

Figure 2. BglII-BclI restriction analysis of chromosomal DNA from gpt transformants. The structure of the SV40 early region in COS1 cells and the predicted structure in transformants derived from homologous integrations of plasmids $\Delta 1$ and $\Delta 2$ are shown together with the Southern blot analysis. Probes are indicated by open bars (top). Putative homologous integrants are marked with an asterisk (bottom). Numbers at the sides refer to the sizes of marker DNAs derived for restriction digest of $\lambda$ phage or plasmid DNA. $(\Delta 1-D)$ Putative homologous recombinant derived from an earlier experiment. 


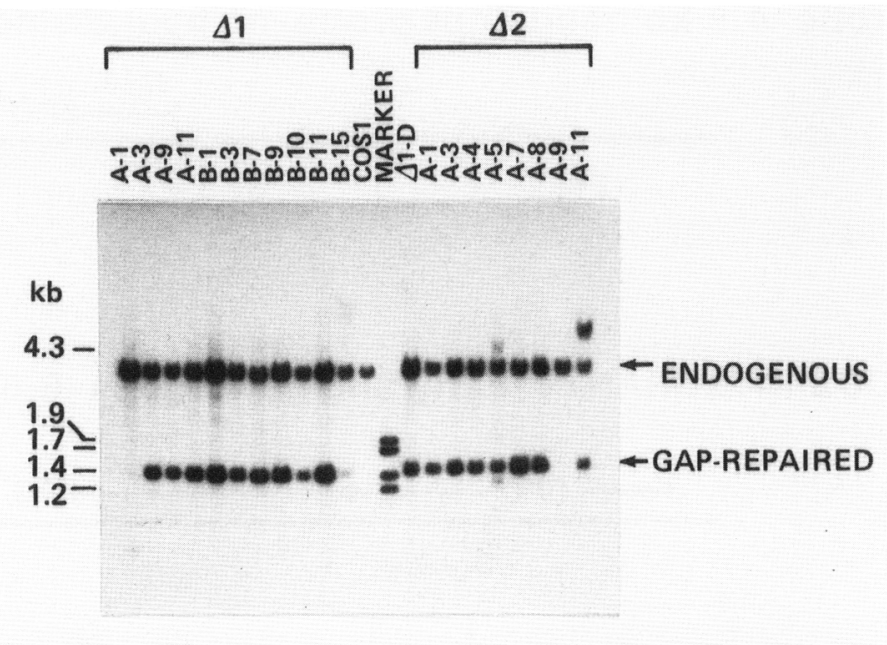

$\Delta 1$ and $\Delta 2$

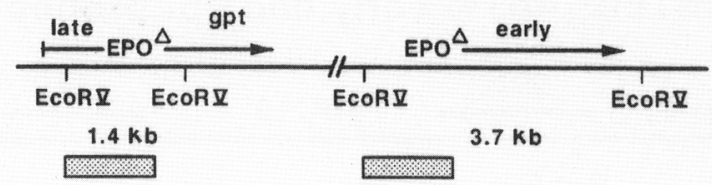

Figure 3. EcoRV restriction analysis of the enhancer region of $g p t^{+}$transformants. The probe, indicated by the stippled bar, overlaps the control region of SV40. The sizes of the EcoRV fragments, 1.4 and $3.7 \mathrm{~kb}$, are indicated below the respective fragments.

The ligated DNA was introduced into Escherichia coli, and ampicillin-resistant clones were isolated. Plasmid DNA prepared from these clones was cleaved with several restriction endounucleases, each yielding patterns completely consistent with the structure shown in Figure 1 (data not shown). Furthermore, as expected, COS1 cells transfected with plasmid DNA derived from one of the clones expressed gpt as efficiently as those that had been transfected with a plasmid containing a complete enhancer-promoter gpt cassette (plasmid 2E), as judged by in vitro enzyme assays. Moreover, after transfection of the $\mathrm{T}$ antigen negative parent cells of COS1 (CV1) with the recovered plasmid DNA, the nuclei in a large percentage of the cells became $\mathrm{T}$ antigen positive, as judged by fluorescent staining with anti-T antigen antibodies.

\section{Conditions affecting the efficiency of detecting homologous integrations}

According to the double-strand break repair model, the enhancer deletion at the gap would be repaired by a gene conversion initiated by invasion of the ends of the transfecting DNA at homologous sequences in the chromosome. This interpretation implies that ends at or near the enhancer deletion are essential for promoting homologous integrations. An additional prediction for such a gap repair process is the necessity for homology between sequences at or near the ends of the transfecting DNAs and those in the chromosomal target. Both of these expectations have been examined in the following experiments.
To assess the necessity for a double-strand break within homology, plasmid $\Delta 2$ was introduced into the COS1 cells with and without the double-strand break at the enhancer deletion or with a break in the pUC19 sequence, a region of nonhomology (Table 2). Nine out of $30 \mathrm{gpt}^{+}$transformants obtained after transfection with

Table 2. Double-strand breaks within homology promote homologous integration of a defective gpt plasmid

\begin{tabular}{lcc}
\hline $\begin{array}{l}\text { Inpuit } \\
\text { DNA }\end{array}$ & $\begin{array}{c}\text { Transformation } \\
\text { efficiency }\end{array}$ & $\begin{array}{c}\text { Homologous } \\
\text { recombinants }\end{array}$ \\
\hline$\Delta 2$ (cut in homology) & $1.5 \times 10^{-5}$ & $9 / 30$ \\
$\Delta 2$ (uncut) & $3 \times 10^{-5}$ & $1^{*} / 20$ \\
$\begin{array}{l}\Delta 2 \text { (cut in nonhomology) } \\
2 \mathrm{E} \text { (cut in homology) }\end{array}$ & $4 \times 10^{-5}$ & $0 / 16$ \\
\hline
\end{tabular}

Input DNA was prepared as follows prior to transfection: $\Delta 2$ (cut in homology), $\triangle 2$ DNA cleaved at the SacI site within the enhancer deletion; $\Delta 2$ (uncut), DNA not cleaved; $\Delta 2$ (cut in nonhomologyl, DNA cleaved at the XmnI site within pUCl9 sequences, which is located about $1160 \mathrm{bp}$ from the start of homology between the plasmid and chromosome; and 2E (cut in homologyl, 2E DNA cleaved at the NaeI site located at the enhancer region. ( ${ }^{*}$ ) A homologous recombinant that may have arisen by a double crossover of the input plasmid. The data for $\Delta 2$ (cut in homology) were derived from three independent platings of cells; all others were derived from two platings. 
plasmid $\Delta 2$ that had been cut at the enhancer deletion were the result of homologous integrations. But when the transfected DNA was not cut, only 1 out of $20 \mathrm{gpt}^{+}$ transformants arose by a homologous recombination. The structure of the single recombinant was anomalous. It can be explained by a double crossover involving a dimer of the transfecting plasmid and the target site, one crossover occurring within the PO region and the other at the intron sequence located downstream of gpt (data not shown). When the break in the transfected $\Delta 2$ plasmid was made in a nonhomologous sequence, no homologous integrants were detected among $16 \mathrm{gpt}^{+}$ transformants analyzed.

We next sought to determine whether increasing the homology between the plasmid sequences flanking the gap and the chromosomal target would alter the relative frequency of homologous integrants. For this purpose, we constructed plasmid $\Delta 3$, which has a segment of the early region sequence downstream of PO (Fig. 1B). In an attempt to reduce the frequency of nonhomologous $g p t^{+}$ transformants, the PO sequence was removed in plasmids $\Delta 4$ and $\Delta 5$ (Fig. 1B), but the same early region segment used in plasmid $\Delta 3$ was in place upstream of gpt. Thus, whereas plasmids $\Delta 1$ and $\Delta 2$ generate monocistronic mRNAs for translation of $g p t$, plasmids $\Delta 3, \Delta 4$, and $\Delta 5$ are predicted to yield dicistronic mRNAs; in the latter three plasmids, translation of their early mRNAs would begin at the AUG initiating the $T$ antigen-coding sequences, presumably terminate upstream of $g p t$, and then reinitiate at the start codon for $g p t$.

Linear DNAs derived from each of these three plasmids, as well as an enhancer-containing derivative of $\Delta 3$, plasmid $3 \mathrm{E}$, were transfected into COSl cells and $g p t^{+}$transformants were recovered (Table 1B). The transformation efficiency with plasmid $3 \mathrm{E}$ is about onethird that of plasmid $2 \mathrm{E}$, suggesting that gpt expression from the putative dicistronic mRNA is somewhat less efficient, a conclusion that is consistent with other studies (Peabody and Berg 1986; D. Strehlow, pers. comm.) and was confirmed by assays of gpt activity in extracts from cells transiently transfected with these plasmids (data not shown). The transformation efficiency with the plasmids $\Delta 3, \Delta 4$, and $\Delta 5$ was $\sim 10$ times lower than with $3 \mathrm{E}$ but only slightly higher than with plasmids $\Delta 1$ and $\Delta 2$. The Southern blot analyses of the DNA from the $g p t^{+}$clones showed that $11-24 \%$ of the transformants arose by homologous insertions at the target sequence. The results suggest that extending the 194-bp homology from the PO end by $613 \mathrm{bp}$ or removing the PO region had little effect on the relative frequency of homologous recombinations.

The rationale for the design of our experiments was that homologous integrations will be efficiently distinguished from a large background of nonhomologous events if the selectable marker is transcriptionally defective. Consequently, nonhomologous integrations of the transfecting plasmids would rarely lead to a $g p t^{+}$ phenotype, whereas homologous integrations would activate transcription of the gpt gene and create a selectable phenotype. However, we wondered whether the large fraction of homologous recombinants was due instead to some other factor, possibly sequences around the SV40 enhancer region acting as a hotspot for homologous recombination. To address this question, we attempted to determine the frequency of homologous integration of plasmid $2 \mathrm{E}$, which is identical to plasmid $\Delta 2$, except that it contains the enhancer sequence. Plasmid 2E was cleaved near the enhancer, transfected into COS1 cells, $g p t^{+}$transformants were recovered as before, and Southern blot analysis of their chromosomal DNA was carried out as described in Figure 2. As expected, the overall frequency of gpt transformation was 100 -fold higher with plasmid $2 \mathrm{E}$ than with $\Delta 2$. Moreover, Southern blot analysis of 35 clones showed variable-sized bands when the blots were hybridized with the gpt probe, but none had an altered target site (Table 2). The difference in transformation frequency between plasmids $2 \mathrm{E}$ and $\Delta 2$, together with the failure to detect homologous recombinants with plasmid $2 \mathrm{E}$, indicates that there was $\sim 100$-fold enrichment for homologous recombinants when the enhancer was deleted. Because one-third of the $g p t^{+}$clones obtained with cleaved plasmid $\Delta 2$ were homologous integrants, we surmise that $\sim 300 \mathrm{gpt}^{+}$transformants produced with plasmid $2 \mathrm{E}$ would have to be screened to find a homologous integrant. We conclude that the SV40 enhancer is not a hotspot for recombination and that our experimental design will have general applications for gene targeting in mammalian cells.

\section{Secondary recombinations at the integration site}

Some of the homologous recombinants appear to be unstable, segregating clones with altered sequence arrangements at the integration site. Such rearrangements were intimated by the occurrence of faint bands of $\sim 3 \mathrm{~kb}$ in Southern blots of BgIII-BcII endonuclease digests (see Fig. 2, e.g., clones $\Delta 1 \mathrm{~B}-1, \Delta 1 \mathrm{~B}-11$, and $\Delta 2 \mathrm{~A}-7)$. To explore the origin of this band, clone $\Delta 1 \mathrm{~B}-1$ was subcloned in medium containing mycophenolic acid. DNA from 17 subclones was subjected to Southern blot analysis using the gpt probe (Fig. 4A). Twelve of the band patterns were identical to those of $\triangle 1 \mathrm{~B}-1$, but the remaining five were different. Two of the DNAs (from subclones 3 and 13) yielded a $2.8-\mathrm{kb}$ fragment, and two others (from subclones 6 and 12) produced a 3.1 -kb fragment. Considering that the integrated plasmid and target sequences share homology in their respective intron segments 1610 $\mathrm{bp})$ and polyadenylation signals $(237 \mathrm{bp})$, we surmised that either intrachromosomal (Fig. 4B) or sister chromatid recombinations between the direct repeats could account for the new fragments. Such recombinations would result in a partial deletion of the early region in these subclones. (Recombination between a third repeat, the late region through EPO ${ }^{\Delta}$, would eliminate the gpt gene; therefore, the resultant subclones would not have survived the selection conditions used to propagate the subclones.) BgIII-BclI endonuclease digestion would yield a $2.8-\mathrm{kb}$ fragment from a recombination involving the intron repeat and a $3.1-\mathrm{kb}$ fragment from a recombi- 


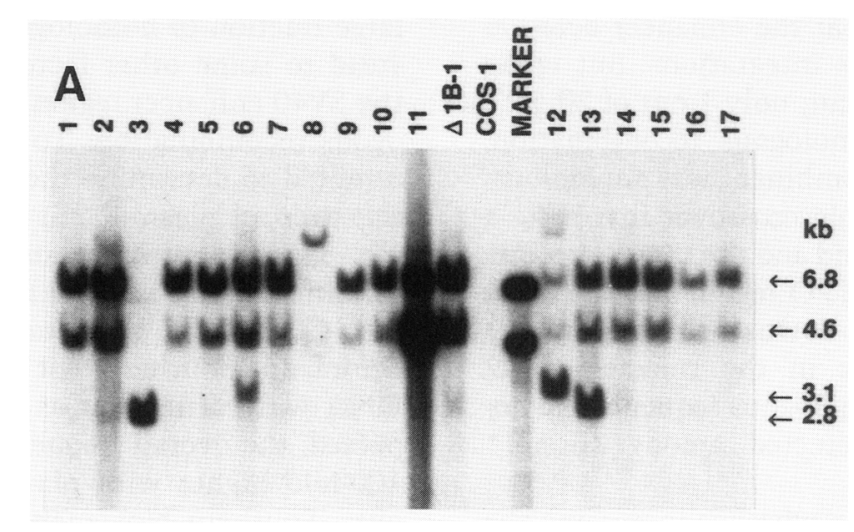

B
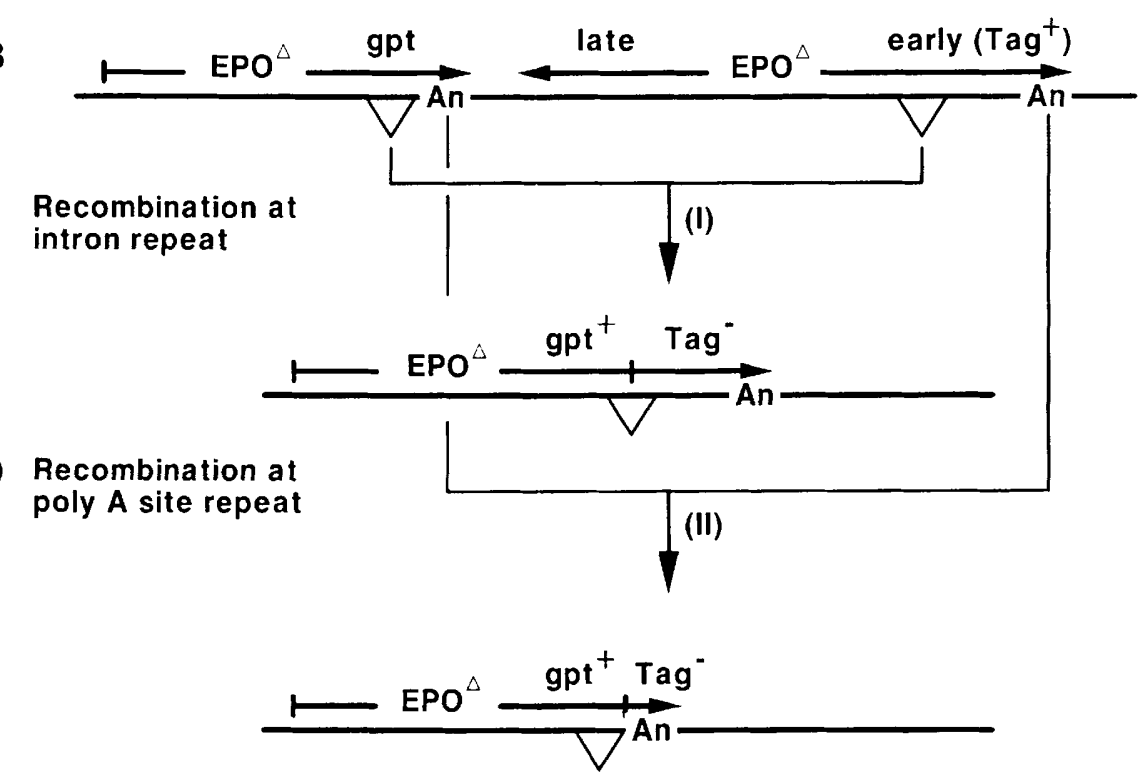

Figure 4. Secondary recombinations at the integration site. $(A)$ Cells from clone $\Delta 1 B-1$ were grown for 6 days after the chromosomal DNA preparation used in the Southern blot shown in Fig. 2 and in the control lane here $(\Delta 1 B-1)$. Subclones $1-17$ were grown for an additional 24 days in gpt selective media prior to DNA isolation. BgIII-BclI restriction and Southern blot analysis was performed as in Fig. 2, using the gpt probe. $(B)$ Possible intrachromosomal recombination at the direct repeats present after homologous integration of plasmid $\Delta 1$. The structure of the integration site in clone $\Delta 1 \mathrm{~B}-1$ is predicted to be similar to that diagramed on the top line, except that an additional tandem copy of plasmid $\Delta 1$ probably exists at that site. An additional unlinked copy of the early region (without gpt integration) is also present. Subclones 3 and 13 conform to the structure indicated after recombination $\mathrm{I}$; subclones 6 and 12 conform to the structure indicated after recombination II.

nation between the polyadenylation repeat. The origin of the larger fragment in subclone 8 remains unexplained.

Note that the $4.6-$ and $6.8-\mathrm{kb}$ bands characteristic of the original $\Delta 1 \mathrm{~B}-1$ homologous recombinant clone are absent from the pattern of subclone 3 and substantially diminished in subclone 12; in contrast, the two parental bands are unchanged in the putative rearrangements of subclones 6 and 13. We presume that the heterogeneity of the restriction patterns is a reflection of when the rearrangement occurred in the growth of the subclone, or of the number of copies of the recombinant sequence that existed at the time of the rearrangement. In all of the subclones, hybridization of the Southern blots with the early region probe confirmed the presence of at least one complete copy of the early region (data not shown). Those subclones that have undergone a deletion at the integration site have retained the second copy of the early region present in the original clone (apparent by the $4.8-\mathrm{kb}$ band in Fig. 2).

The clones that appear to undergo secondary recombinations at high frequency at the integration site are those with tandem integrations of the transfected plasmid. We presume that deletions also occur in the clones with single integrations, albeit at a reduced level. In those instances when it is desirable to achieve deletions in a target gene, a second marker whose loss is selectable can be placed in the transfecting plasmid so as to be positioned between direct repeats at the integration site. 


\section{Discussion}

Our experiments show that homologous integration in mammalian cells can be efficiently selected if, as a result of the recombination, the genomic target provides an essential element for the expression of an otherwise nonfunctional transfected gene. In the present instance, the recombination event provides an enhancer from an integrated SV40 early region to a gpt gene lacking the enhancer in its SV40 early promoter. With this experimental design, homologous integration occurred in 25 of $57 \mathrm{gpt}^{+}$clones $(44 \%)$ from six independent transfections with plasmids $\Delta 1$ and $\Delta 2$; moreover, homologous integrations following transfections with related plasmids $(\Delta 3-\Delta 5)$ ranged between $11 \%$ and $24 \%$. Considering the failure to detect homologous integrants among the $g p t^{+}$ transformants when the COS1 cells were transfected with an analogous linear plasmid containing the enhancer (plasmid 2E), we estimate that there is a 100 -fold increase in the probability of detecting and recovering homologous integrants by requiring repair of the enhancer deletion. Based on the combined results with plasmids $\Delta 2$ and $2 \mathrm{E}$ (Table 2), our estimate is that there is $\sim 1$ homologous recombinant to 300 nonhomologous integrants, which is within the range seen in other mammalian recombination systems (Smith and Berg 1984; Lin et al. 1985; Thomas et al. 1986; Doetschman et al. 1987; Song et al. 1987; Thomas and Capecchi 1987). It seems unlikely, therefore, that the SV40 sequences provide a hotspot for recombination or that the expression of $\mathrm{T}$ antigen greatly facilitates such recombination events.

Our findings allow parallels to be drawn between yeast integration events that are proposed to occur by the double-strand gap repair mechanism (Szostak et al. 1983 ) and mammalian integration events. Gapped linear DNAs with ends at or near the homologous sequence to be repaired were the most efficient substrates for homologous integration. Sealing the DNA's ends or moving the ends to regions of nonhomology with the chromosomal target virtually eliminated the recombinational insertion. This is the first clear demonstration of an enhancement of mammalian homologous integration by a double-strand break within a region of homology. Interand intraplasmid recombination has also been shown to be stimulated by double-strand breaks; however, it is presumed to occur predominantly through a different mechanism involving the nonconservative joining of free ends (Lin et al. 1984; Anderson and Eliason 1986; Chakrabarti and Seidman 1986).

Figure 5 illustrates how features of our system for gene targeting can be adapted to other systems. Both of the displayed examples rely on the selectable marker acquiring an active promoter element from the homologous target upon integration. In the top example, a gene fragment within the target's transcription unit provides the homology so that the target gene forfeits its promoter segment to the gpt gene upon integration. The bottom example recapitulates the integration scheme described in this paper in which the homology flanks the

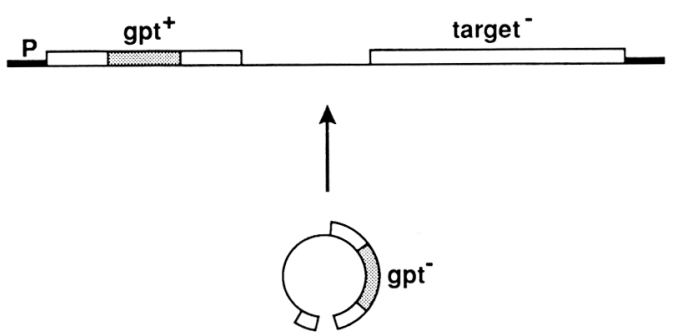

Genomic Site target $^{+}$
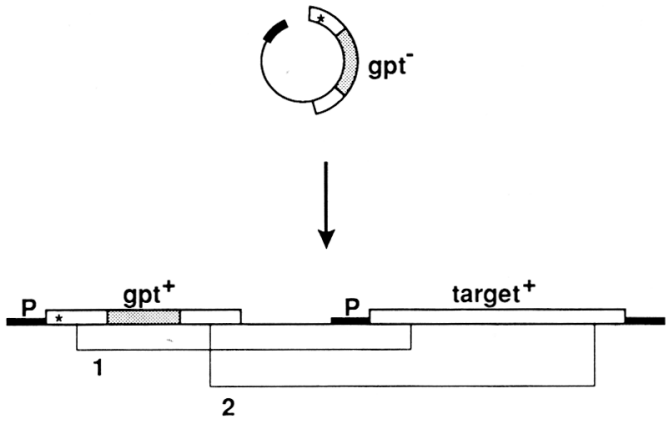

Figure 5. Targeting of transfected DNA to chromosomal loci in mammalian cells with (top) or without (bottom) disruption of the target gene. The transfected DNA (broken circles) includes a defective selectable marker, in this case, the gpt gene, and is cleaved prior to transfection (indicated by gaps in the circles|. The marker is fused to sequences derived from the target gene (open bars) such that a homologous integration results in the activation of the marker. (Top) The homology is internal to the transcription unit, resulting in a disruption of the target gene. Here, the upstream promoter elements $(P)$ become separated from the target after integration. (Bottom) The homology flanks the promoter (solid and open bars). Gene conversion during gap repair results in a duplication of the promoter such that the target is not disrupted. Subsequent recombination events are shown, which can lead to mutation (1) or loss (2) of the target gene. $\left({ }^{*}\right)$ A mutation to be introduced into the target gene.

promoter so that the target gene is not disrupted. However, sequence homologies between the integrated DNA and the target can lead to replacement of the original target sequence by a modified form (recombination 1) or to elimination of the target sequence (recombination 2). The frequency of the former event, which creates mutations in the target and results in loss of the gpt gene, can be increased by selection in appropriate media. Although the defect in the gpt marker shown here is in promoter elements, it may be possible to integrate into unexpressed loci using a marker defective at some other position, e.g., intron sequences or a polyadenylation site. 


\section{Methods \\ Cell growth and transfection}

COS1 cells were cultured in Dulbecco's modified Eagle's minimal essential medium (GIBCO), containing $5 \%$ fetal calf serum and penicillin/streptomycin. Cells were seeded 1 day prior to transfection at $\sim 7 \times 10^{5}$ cells per $10-\mathrm{cm}$ dish. The calcium phosphate coprecipitation method was used as described previously (Jasin et al. 1985) with $20 \mu \mathrm{g}$ of plasmid DNA. Transformants were selected according to Mulligan and Berg (1981), except that $12.5 \mu \mathrm{g} / \mathrm{ml}$ mycophenolic acid, $1 \mu \mathrm{g} / \mathrm{ml} \mathrm{ami-}$ nopterin, $5 \mu \mathrm{g} / \mathrm{ml}$ glycine, and $100 \mu \mathrm{g} / \mathrm{ml}$ xanthine were used.

\section{Recombinant DNA constructs and techniques}

All DNA constructs were made by standard recombinant DNA techniques under conditions conforming to the National Institutes of Health Guidelines for Recombinant DNA Research. The integrating plasmids $\Delta \mathrm{l}-\Delta 5$ each consist of the gpt gene linked to various SV40 sequences for homology with the genomic target but are deleted for enhancer or enhancer/promoter sequences. Plasmids $2 \mathrm{E}$ and $3 \mathrm{E}$ are similar to the integrating plasmids $\Delta 2$ and $\Delta 3$, respectively, except that they contain a complete transcription enhancer/promoter region. Unless otherwise indicated, $\Delta 2, \Delta 3, \Delta 4$ were linearized with SacI, which cleaves at the enhancer deletion, prior to transfection. Similarly, $\Delta \mathrm{l}$ and $\Delta 5$ were cleaved with SacI-SphI and SacI-SalI, respectively, to liberate the gpt gene linked to the late region from pUC19 sequences, although this fragment was not purified prior to transfection. Plasmid $\Delta \mathrm{l}$ was constructed in two steps. In the first, the 2.2-kb KpnI-BamHI SV40 late region segment from plasmid pET-1 (Weber et al. 1984) was subcloned into pUC19. Subsequently, the 1.9-kb BamHI-HindIII gpt fragment from pSV2gpt and the 194-bp HindIII-SphI SV40 promoter segment from plasmid 6-1 (Gluzman et al. 1980) were ligated to a BamHI-SphI digest of the above subclone. The promoter segment contains a 6-bp deletion eliminating the BgII site. Integrating plasmid $\Delta 2$ was constructed through an intermediate plasmid that has the 1.5-kb EcoRI-SacI late region segment from $\Delta 1$ cloned into pUC19. This intermediate plasmid was cleaved with SmaI and PstI and ligated to the 2.6$\mathrm{kb} S p h \mathrm{I}-P s t \mathrm{I} g p t$ fragment from $\Delta \mathrm{l}$. Prior to this ligation, the $S p h I$ end was trimmed by T4 DNA polymerase to create a blunt end capable of being joined to the SmaI end of the intermediate plasmid. Plasmid $\Delta 3$ was constructed in two steps, beginning with the intermediate plasmid in the $\Delta 2$ construction. This intermediate was cleaved with $P$ stI and BamHI and ligated to the 678-bp HpaI-PstI fragment from $\Delta 1$ containing the SV40 polyadenylation site and the 930-bp ApaI-BglII gpt fragment, also from $\Delta 1$. The resultant plasmid was cleaved with $S m a I$ and ligated to the 807-bp SphI-PflMI early region fragment from plasmid 6-1. For these two constructions, the ApaI, SphI, and PflMI ends were made blunt by T4 DNA polymerase. The promoter/origin deletion version of $\Delta 3$, plasmid $\Delta 4$, was constructed by cleaving the intermediate plasmid for $\Delta 2$ with $X b a I$ and PstI and inserting the $2.2-\mathrm{kb}$ AvrII-Pst gpt fragment from $\Delta 3$. Plasmid $\Delta 5$ was constructed by ligating SacI-BamHIcleaved pUC19 with the $1.7-\mathrm{kb}$ AvrII-BamHI gpt fragment from $\Delta 3$ and the $2.2-\mathrm{kb}$ SacI-BamHI late region fragment from $\Delta 1$. Plasmids $2 \mathrm{E}$ and $3 \mathrm{E}$ were constructed by ligating the large AvrII fragment of plasmids $\Delta 2$ and $\Delta 3$, respectively, with the 1128-bp AvrII enhancer fragment from plasmid 6-1.

Southern analysis (Southern 1975) was performed essentially according to Maniatis et al. (1982), using modifications suggested by the manufacturer of GeneScreenPlus (DuPont). BglII-BcII-restricted chromosomal DNA $(5 \mu \mathrm{g})$ was fraction- ated on an $0.8 \%$ agarose gel. The early region probe is the $1038-$ bp NdeI-BcII fragment from SV40; the gpt probe is the 930-bp ApaI-BgIII fragment from pSV2gpt. EcoRV-cleaved chromosomal DNA was electrophoresed on a $1.0 \%$ agarose gel and probed, after transfer, with an 840-bp EcoRV-HindIII fragment overlapping the control region of SV40.

\section{Acknowledgments}

This work was initiated in the laboratory of Walter Schaffner (Zürich). The gift of plasmid 6-1 from Yakov Gluzman (Lederle Labs) is gratefully acknowledged. We thank Janet Braam, Susanna Lewis, and Richard Mann for extensive comments on the manuscript. M.J. was a fellow of the Jane Coffin Childs Memorial Fund for Medical Research. This work was supported by the National Institutes of Health.

\section{References}

Anderson, R.A. and S.L. Eliason. 1986. Recombination of homologous DNA fragments transfected into mammalian cells occurs predominantly by terminal pairing. Mol. Cell. Biol. 6: 3246-3252.

Chakrabarti, S. and M.M. Seidman. 1986. Intramolecular recombination between transfected repeated sequences in mammalian cells is nonconservative. Mol. Cell. Biol. 6: $2520-2526$.

Doetschman, T., R.G. Gregg, N. Maeda, M.L. Hooper, D.W. Melton, S. Thompson, and O. Smithies. 1987. Targetted correction of a mutant HPRT gene in mouse embryonic stem cells. Nature 330: $576-578$.

Gluzman, Y. 1981. SV40-transformed simian cells support the replication of early SV40 mutants. Cell 23: 175-182.

Gluzman, Y., J.F. Sambrook, and R.J. Frisque. 1980. Expression of early genes of origin-defective mutants of simian virus 40 . Proc. Natl. Acad. Sci. 77: 3898-3902.

Jasin, M., J. de Villiers, F. Weber, and W. Schaffner. 1985. High frequency of homologous recombination in mammalian cells between endogenous and introduced SV40 genomes. Cell 43: 695-703.

Lin, F.-L., K. Sperle, and N. Sternberg. 1984. Model for homologous recombination during transfer of DNA into mouse $\mathrm{L}$ cells: Role for DNA ends in the recombination process. Mol. Cell. Biol. 4: 1020-1034.

- 1985. Recombination in mouse L cells between DNA introduced into cells and homologous chromosomal sequences. Proc. Natl. Acad. Sci. 82: 1391-1395.

Maniatis, T., E.F. Fritsch, and J. Sambrook. 1982. Molecular cloning: A laboratory manual. Cold Spring Harbor Laboratory, Cold Spring Harbor, New York.

Mulligan, R.C. and P. Berg. 1980. Expression of a bacterial gene in mammalian cells. Science 209: 1422-1427.

. 1981. Selection for animal cells that express the $E$. coli gene coding for xanthine-guanine phosphoribosyltransferase. Proc. Natl. Acad. Sci. 78: 2072-2076.

Orr-Weaver, T.L. and J.W. Szostak. 1983. Yeast recombination: The association between double-strand gap repair and crossing-over. Proc. Natl. Acad. Sci. 80: 4417-4421.

Peabody, D.S. and P. Berg. 1986. Termination-reinitiation occurs in the translation of mammalian cell mRNAs. Mol. Cell. Biol. 6: 2695-2703.

Scherer, S. and R. Davis. 1979. Replacement of chromosomal segments with altered DNA sequences constructed in vitro. Proc. Natl. Acad. Sci. 76: 4951-4955.

Smith, A.J.H. and P. Berg. 1984. Homologous recombination be- 
tween defective neo genes in mouse $3 \mathrm{~T} 6$ cells. Cold Spring Harbor Symp. Quant. Biol. 49: 171-181.

Smithies, O., R.G. Gregg, S.S. Boggs, M.A. Koralewski, and R.S. Kucherlapati. 1985. Insertion of DNA sequences into the human chromosome $\beta$-globin locus by homologous recombination. Nature 317: 230-234.

Song, K.Y., F. Schwartz, N. Maeda, O. Smithies, and R.S. Kucherlapati. 1987. Accurate modification of a chromosomal plasmid by homologous recombination in human cells. Proc. Natl. Acad. Sci. 84: 6820-6824.

Southern, E.M. 1975. Detection of specific sequences among DNA fragments separated by gel electrophoresis. J. Mol. Biol. 98: 503-517.

Szostak, J.W., T.L. Orr-Weaver, R.J. Rothstein, and F.W. Stahl. 1983. The double-strand-break repair model for recombination. Cell 33: 25-35.

Thomas, K.R. and M.R. Capecchi. 1987. Site-directed mutagenesis by gene targeting in mouse embryo-derived stem cells. Cell 51: 503-512.

Thomas, K.R., K.R. Folger, and M.R. Capecchi. 1986. High frequency targeting of genes to specific sites in the mammalian genome. Cell 44: 419-428.

Weber, F., J. de Villiers, and W. Schaffner. 1984. An SV40 'enhancer trap' incorporates exogenous enhancers or generates enhancers from its own sequences. Cell 36: 983--992. 


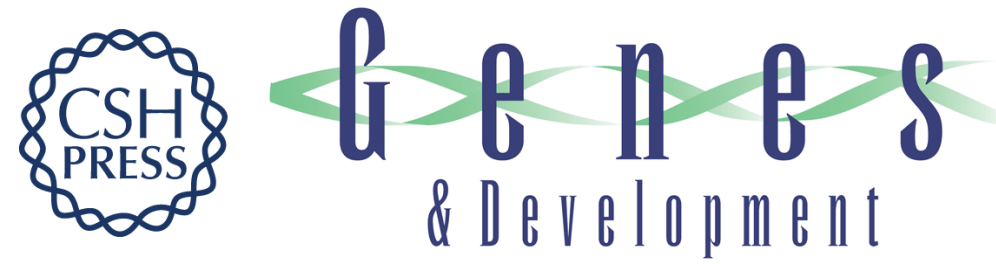

\section{Homologous integration in mammalian cells without target gene selection.}

$M$ Jasin and P Berg

Genes Dev. 1988, 2:

Access the most recent version at doi:10.1101/gad.2.11.1353

References This article cites 21 articles, 12 of which can be accessed free at:

http://genesdev.cshlp.org/content/2/11/1353.full.html\#ref-list-1

License

Email Alerting

Service

Receive free email alerts when new articles cite this article - sign up in the box at the top right corner of the article or click here.

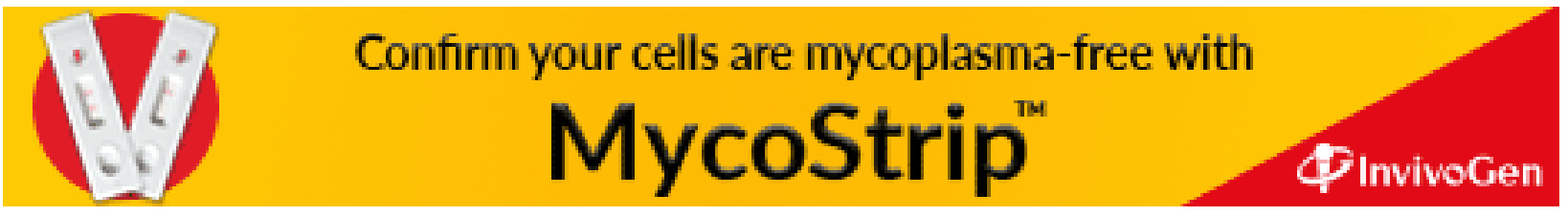

